\title{
Relative Aortic Blood Pressure Using 4D Flow MRI: Associations with Age and Aortic Tapering
}

\author{
Kevin Bouaou ${ }^{1}$, Ioannis Bargiotas ${ }^{1}$, Damian Craiem ${ }^{3}$, Gilles Soulat ${ }^{2}$, Thomas Dietenbeck ${ }^{1}$, Sophia \\ Houriez--Gombaud-Saintonge ${ }^{1}$, Alain De Cesare ${ }^{1}$, Umit Gencer ${ }^{2}$, Alain Giron ${ }^{1}$, Alban Redheuil ${ }^{1}$, \\ Didier Lucor $^{4}$, Elie Mousseaux ${ }^{2}$, Nadjia Kachenoura ${ }^{1}$. \\ ${ }^{1}$ Sorbonne Universités, UPMC Univ Paris 06, INSERM 1146, CNRS 7371, Laboratoire d'Imagerie \\ Biomédicale, Paris, France \\ ${ }^{2}$ Hôpital Européen Georges Pompidou, Paris, France \\ ${ }^{3}$ Universidad Favaloro-CONICET, IMeTTyB, Buenos Aires, Argentina \\ ${ }^{4}$ LIMSI, CNRS, Université Paris-Saclay, Orsay, France
}

\begin{abstract}
MRI $4 D$ flow velocity is used to evaluate relative blood pressures in the aorta throughout the cardiac cycle. This $(3 D+t)$ pressure mapping is used to assess: 1) relationship between trans-aortic pressure gradient with age and aortic tapering (proximal to distal narrowing in lumen area), 2) effect of temporal resolution.

We studied 47 healthy subjects (49 \pm 17.6 years, 24 men) who underwent $4 D$ flow MRI, among them 20 were reconstructed in 20 and 50 phases per cardiac cycle. Pressure gradients maps were estimated from velocity fields using the Navier-Stokes equations. Relative pressures were calculated using an iterative algorithm while considering a zero pressure at the aortic valve.

Distal (DA) to proximal (PA) aortic pressure gradient decreased with age $(r=0.60, p<0.05)$ and was inversely related to DA/PA areas ratio $(r=0.35, p<0.05)$. Such result is in line with the physiological evidence indicating that in aging DA area tends to equalize with PA area lowering the DA to PA pressure gradient. Peak systolic pressure was higher when considering the 50 phases data. Relative pressures calculated from $4 D$ flow within the whole aortic volume through time are consistent with prior physiological knowledge as demonstrated by their variations with age and with aortic geometry.
\end{abstract}

\section{Introduction}

Aortic pressure gradients are useful in characterizing diseases such as valvular stenosis and aortic coarctation [1]. Although catheterization is the gold standard for the measurement of local aortic pressures, its invasive nature limits its usefulness in clinical routine. Alternatively, applanation tonometry has been proposed for a noninvasive evaluation of central arterial pressure variations through time. Although it is well accepted that pressure distribution varies locally throughout the aorta, none of the aforementioned methods can provide the exact spatial pressures distribution within the aorta.

It is acknowledged that pressure gradient is directly related to fluid acceleration [4]. Thus, velocities can help estimating noninvasively aortic pressure gradients. Several formulations can be used to evaluate pressure gradients non-invasively in the central cardiovascular system. They all rely on models based/derived from the Navier-Stokes equations. For instance, the simplified Bernoulli equation uses the maximal velocity while neglecting the temporal acceleration [1].

A more elaborate approach is based on the resolution of a modified version of the Navier-Stokes equations when one holds the velocity field thanks to $4 \mathrm{D}$ flow MRI. In this case, it is in general assumed that blood is incompressible, laminar and Newtonian [1,3,4]. Using such approach, spatio-temporal pressure maps can be computed by: 1) spatially integrating pressure gradients obtained from Navier-Stokes equations, while assuming a zero pressure at the level of aortic valve, 2) applying an iterative refinement resulting in smooth relative pressures within the segmented aorta through time [3]. Several studies have evaluated this pressure map on small populations including either healthy subjects or patients suffering from vascular disease such as coarctation and aneurysm. However, effect of proximal (PA) to distal (DA) aortic tapering with aging and time resolution remains not fully investigated.

Accordingly, our objectives were to: 1) use 4D flow MRI along with Navier-Stokes model-equations in order to generate volumetric relative pressures $(3 \mathrm{D}+\mathrm{t}), 2)$ evaluate effect of temporal resolution on peak systolic relative pressure as well as on distal to proximal aortic pressure gradients, and 3) evaluate the relationship between trans-aortic pressure gradient with age and aortic tapering (proximal to distal change in lumen area). 


\section{Material and Methods}

\subsection{Navier-Stokes Equation}

The Navier-Stokes equations govern the motion of fluids through a balance of inertial, viscous, external and pressure forces. In particular, given a 3D velocity field $(v)$ of a viscous and incompressible fluid, the Navier-Stokes momentum contribution equation can be used to deduce the unknown pressure gradient $(\nabla P)$ :

$$
\nabla P=-\rho(\partial v / \partial t+v \cdot \nabla v)+\mu \nabla^{2} v+\rho g(1)
$$

where $\rho$ is the fluid density ( $\rho_{\text {blood }}=1060 \mathrm{~kg} \cdot \mathrm{m}^{-3}$ ), $\mu$ is the dynamic viscosity $\left(\mu_{\text {blood }}=0.0035 \mathrm{~kg} \cdot \mathrm{m}^{-1} \cdot \mathrm{s}^{-1}\right)$ and $\mathrm{g}$ is the gravitational force exerted on the blood. Navier-Stokes equation balance several flow contributions: temporal inertia $\partial v / \partial t$, convective inertia $v \cdot \nabla v$, viscous resistance $\mu \nabla^{2} v$, and body force term $\rho g$. Viscous resistance is significant near the walls $(<1 \mathrm{~mm}$ in the aorta) and can therefore be neglected. The body force is also neglected since the subject was studied in a supine position [4].

\subsection{Population and data acquisition}

Time-resolved 3D encoded phase contrast MRI was performed on 47 healthy subjects (age $=49.5 \pm 18$ years, 24 women) without overt cardiovascular disease.

MRI was performed on a 3T imaging system (GE Medical Systems, Milwaukee, WI) with cardiac phasedarray coil, ECG-gated and respiratory motion control using the following scan parameters: echo time $=1.7 \mathrm{~ms}$, repetition time $=4.3-4.4 \mathrm{~ms}$, flip angle $=15^{\circ}$, spatial resolution $=1 \times 1.48 \times 2.38 \mathrm{~mm}^{3}$, and velocity encoding was $250 \mathrm{~cm} / \mathrm{s}$ in all directions. All subject's data were reconstructed with 50 phases per cardiac cycle and a subgroup of 20 subjects, randomly selected, were reconstructed with 20 phases (no significant differences). Reconstructions (20 and 50 phases) were done on the same dataset with different interpolation and view sharing through time within the $\mathrm{k}$ space. Baseline characteristics, central blood pressures, augmentation index and pulse pressures were collected.

\subsection{MRI 4D flow data preprocessing}

Algorithms and user interface were written in Matlab 2016 (The Mathworks, Natick, MA).

Prior to the estimation of pressure gradient, phase offset and phase wrapping were corrected according to the 4D flow MRI clinical applications guidelines [5]. Moreover, a delineation of the aorta in the $4 \mathrm{D}$ volume was performed, since such step is crucial to estimate the pressures within the vessel while limiting effect of external noise. To achieve such aortic segmentation a phase contrast MRI angiography (PC-MRA) was first calculated as time average combining velocity fields and magnitude [1], in order to amplify the signal inside the aortic lumen. Then such PC-MRA was used to segment the thoracic aorta with an explicit active contours algorithm [2]. Finally, aortic centreline was extracted.

\subsection{Pressure Map Estimation}

First, the pressure gradient is estimated using NavierStokes equation (1) and the velocity fields within the segmented aorta as explained above. The derivative terms were evaluated thanks to a central difference formula between three consecutive pixels for the convective acceleration term and three consecutive frames for the temporal acceleration term, while considering the $\mathrm{x}, \mathrm{y}$ and $\mathrm{z}$ directions. The summation of the convective and the temporal terms resulted in a 3D pressure gradient map for each time frame.

The Navier-Stokes equation assumes an incompressible fluid, but real conditions in 4D flow data result in noise that do not comply with such hypothesis. To overcome this issue, an iterative method based on the discretization of the pressure Poisson equation $[3,4,6]$ was used. An initial pressure map $P_{i}$ is estimated by propagating the relative pressure starting from a reference point (Figure 1) to the nearest neighbors using NavierStrokes pressure gradient. Afterwards the final relative pressure $P_{f}$ is calculated while verifying that the gradient of $P_{f}$ is as close as possible of the Navier-Stokes pressure gradient evaluated from the velocity fields. This is done through solving the following iterative equation $[3,4,6]$ :

$$
{P_{f}}^{k+1}=(1-\alpha) P_{f}{ }^{k}+\frac{1}{6} \alpha \sum_{i=1}^{6}\left(P_{i}^{k}+\nabla P_{i} \cdot \Delta r_{i}\right)
$$

where $P_{f}{ }^{k}$ is the pressure value at the $\mathrm{k}^{\text {th }}$ iteration, $\mathrm{P}_{\mathrm{i}}$ and $\nabla P_{i}$ were relative pressure and pressure gradient of the six orthogonal neighbors, $\Delta r_{i}$ the voxel size and $\alpha$ a scalar set to $0.5[3,6]$.

The programming approach used to calculate Pi was based on a 3D Euclidian distance map according to the reference point, which defines the path used to browse the aortic volume. Such path is used to calculate the relative pressure in successive voxels by mean integration between central voxel and non-zero neighbors. Such calculation is done only if at least one neighbor has a defined value and a second iteration is performed for the missing voxels. For the subgroup of subjects reconstructed with 20 and 50 phases, relative pressure maps were calculated for both reconstructions resulting in two different pressure maps for each subject.

\subsection{Proximal to distal aortic pressures}

For each subject, six aortic sections perpendicular to 
centerline covering both proximal aorta and distal descending aorta were defined. Aortic cross sectional planes were equally spaced along the centerline starting from the reference point at the aortic valve (Figure 1). For each plane, pressure curves were calculated by averaging the pressure values in a region of interest located around the centerline, while avoiding the noisy borders. Distal to proximal pressure gradient was calculated as the difference in peak systolic relative pressure between plane 6 and plane 1. Similarly, tapering is expressed as the ratio of lumen areas between plane 6 and plane 1. To assess effect of time resolution, peaks systolic relative pressure were estimated for all planes and for both the 50 and 20 phases-derived pressure maps and were compared using the nonparametric Wilcoxon test.

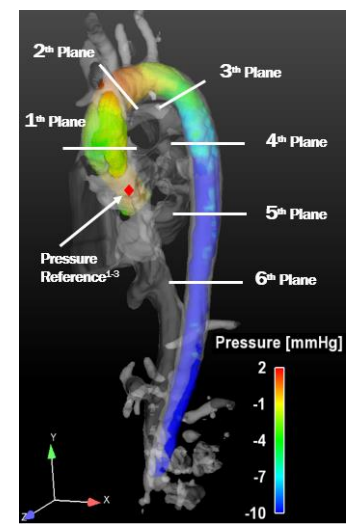

Figure 1.3D relative pressure map and 6 defined planes.

\section{Results}

Baseline characteristics as well as central blood pressures are summarized in Table 1

Table 1. Subjects characteristics, and central blood pressures. BMI: body mass index. SBP/DPB: systolic/diastolic blood pressures

\begin{tabular}{lc}
\hline Parameters & Whole group \\
\hline Age (years) & $49.5 \pm 18$ \\
Sex (men/women) & $23 / 24$ \\
BMI $\left(\mathrm{kg} / \mathrm{m}^{2}\right)$ & $23.6 \pm 2.7$ \\
Heart rate (b.p.m) & $67.3 \pm 12.3$ \\
SBP (mmHg) & $113.1 \pm 13.7$ \\
DBP (mmHg) & $78.8 \pm 9.1$ \\
\hline
\end{tabular}

\subsection{Effect of temporal resolution}

Pressure maps were evaluated for both the 20 and 50 phases reconstructions in a subgroup of 20 subjects. Relative peak systolic pressures were significantly higher for the 50 phases data, as compared to the 20 phases, independent of the considered plane $(\mathrm{p}<0.01$, for all the planes) (Figure 2). Such finding suggests that, the systolic peak might be missed in low time resolution data $(20$ phases).

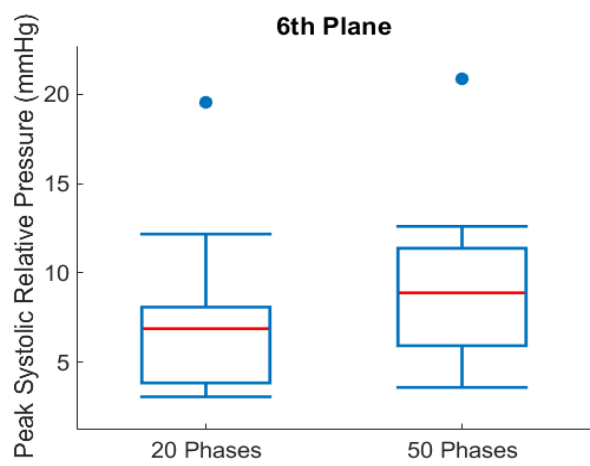

Figure 2: Peak systolic relative pressure averaged over the 20 subjects on plane 6 for 20 and 50 phases data.

\subsection{Pressure variations with age and aortic tapering}

For the 47 studied patients, the distal to proximal peak systolic gradient decreased linearly with age as illustrated in Figure 3.A $(|\mathrm{r}|=0.60, \mathrm{p}<0.05)$. Furthermore, distal to proximal peak systolic gradient decreased with distal to proximal aortic tapering $(|\mathrm{r}|=0.35, \mathrm{p}<0.05)$ as indicated by a negative association between the ratio of distal to proximal peak systolic relative pressures and the ratio of distal to proximal aortic lumen areas (Figure 3.B). Such associations with age are consistent with the physiological knowledge, which indicates that pressures equalizes between central and distal parts of the aorta as distal to proximal aortic lumen areas difference decrease with age.

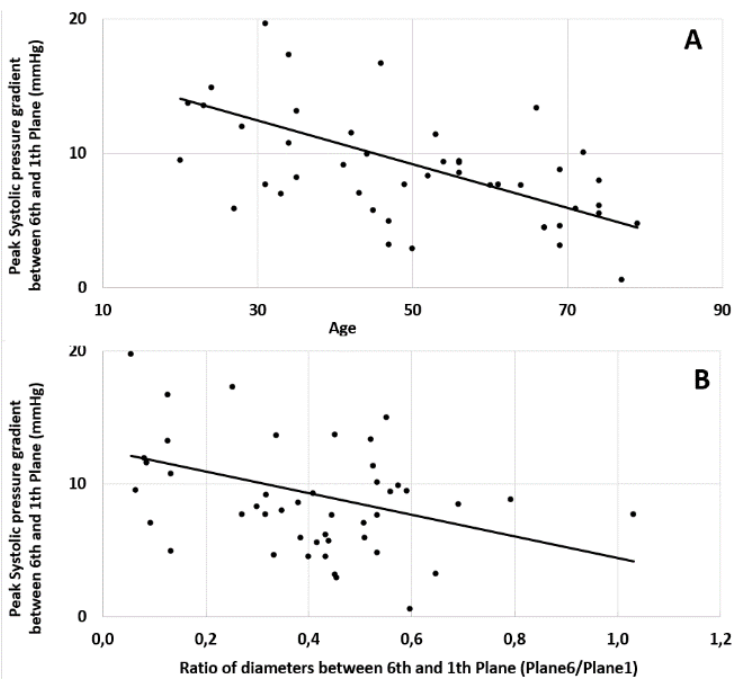

Figure 3. Association of distal to proximal peak systolic pressure gradient with age (A) and areas ratio (B) 


\section{Discussion}

In this work, we demonstrated that $3 \mathrm{D}$ aortic pressure maps relative to a zero pressure in the aortic valve is feasible using 4D flow MRI data in a large group of healthy volunteers. Such estimate was affected by temporal resolution of the MRI data indicating the difficulty to evaluate aortic pressures in low resolution data, which are commonly acquired in the majority of MRI centres performing 4D flow, for shortening the acquisition time. Physiological consistency has been shown through the associations of distal to proximal pressure gradient with age and aortic geometrical tapering.

Previous studies using Navier-Stokes equation and MRI velocity data to evaluate aortic relative pressure have been published $[1,3,6,7]$. Our findings were in agreement with those reported in studies using discretisation of the pressure Poisson equation to evaluate the relative pressure [1]. In such previous studies, relative pressure maps were evaluated in small groups of healthy subjects and for various aortic pathological cases such as aortic aneurysm or coarctation. To the best of our knowledge, our study is the first to assess consistency with age and aortic geometrical tapering and to investigate the effect of temporal resolution. Indeed, since temporal inertia plays a key role in the evaluation of the pressure gradient $[4,7]$, the error due to low temporal resolution of 4D flow MRI should be investigated, as this is one of the major drawbacks of 4D flow MRI. In addition, this temporal resolution influences the evaluation of pressure gradient.

As previously suggested [4], viscous resistance term was neglected in pressure gradient evaluation. Indeed, this term would have amplified the noise, because of its estimation as a second derivative. However, in case of healthy volunteers the aortic radius is large enough to afford neglecting such viscous term.

\section{Conclusion}

Relative pressures calculated from Navier-Stokes equation together with 4D flow MRI within the whole aortic volume through the entire cardiac cycle are consistent with physiological knowledge as demonstrated by their variations with age and with aortic geometrical tapering. Such estimates of aortic pressure may prove its usefulness in the understanding of hemodynamic changes secondary to disease. Also local pressures may be of major usefulness in optimizing the assessment of aortic complication risk with imaging, presently based only on area measurements, shown to be imperfect, since $50 \%$ of patients with aortic dissection have normal sized thoracic aorta (from IRAD International Registry of Acute Aortic Dissections). Indeed, having the inner hemodynamic information in addition to changes in geometry may help to better identify aortic zones at risk of dissection.

\section{Acknowledgement}

We would like to acknowledge the FRM project ING20150532487 for funding a large part of the technical aspects of this study as well as the ECOS-SUD project number A15S04 (France-Argentina) exchange grant for funding a fruitful exchange around 4D flow MRI data processing with D Craiem team.

\section{References}

[1] Bock J, Frydrychowicz A, Lorenz R, et al. In vivo noninvasive $4 \mathrm{D}$ pressure difference mapping in the human aorta: Phantom comparison and application in healthy volunteers and patients. Magn Reson Med 2011; 66: $1079-1088$

[2] Dietenbeck T, Roseenbaum D., Damian C., et al. Semiautomated quantification of aortic arch length and stiffness in CMR using a 3D cylindrical active surface. Proceeding SCMR 2017.

[3] Bargiotas I, Redheuil A, Evin M, et al. Pixel-wise absolute pressures in the aortic arch from 3D MRI velocity data and carotid artery applanation tonometry. In: 2014 36th Annual International Conference of the IEEE Engineering in Medicine and Biology Society. 2014, pp. 5105-5108.

[4] Yang G-Z, Kilner PJ, Wood NB, et al. Computation of flow pressure fields from magnetic resonance velocity mapping. Magn Reson Med 1996; 36: 520-526.

[5] Dyverfeldt $\mathrm{P}$, Bissell $\mathrm{M}$, Barker AJ, et al. 4D flow cardiovascular magnetic resonance consensus statement. J Cardiovasc Magn Reson; 17. Epub ahead of print 10 August 2015. DOI: 10.1186/s12968-015-0174-5.

[6] Tyszka JM, Laidlaw DH, Asa JW, et al. Threedimensional, time-resolved (4D) relative pressure mapping using magnetic resonance imaging. J Magn Reson Imaging 2000; 12: 321-329.

[7] Lamata P, Pitcher A, Krittian S, et al. Aortic Relative Pressure Components Derived from Four-Dimensional Flow Cardiovascular Magnetic Resonance. Magn Reson Med 2014; 72: 1162-1169.

Address for correspondence.

Bouaou Kevin

Faculté de Médecine Pierre et Marie Curie - Site Pitié Salpêtrière- LIB, 91 Bd de l'Hôpital, 75013, Paris, France kevin.bouaou@gmail.com 